\title{
The pentaquark $\Theta^{+}(1540)$ in the string model
}

\author{
B.V. Martemyanov ${ }^{1,2}$, C. Fuchs ${ }^{1}$, Amand Faessler ${ }^{1}$, and M.I. Krivoruchenko ${ }^{1,2}$ \\ 1 Institut für Theoretische Physik, Universität Tübingen,, \\ Auf der Morgenstelle 14,D-72076 Tübingen, Germany \\ 2 Institute for Theoretical and Experimental Physics, \\ B. Cheremushkinskaya 25, 117259 Moscow, Russia
}

(Dated:)

\begin{abstract}
We consider the $\Theta^{+}(1540)$ pentaquark in the string model that correctly reproduces the linear Regge trajectories for the case of orbital excitations of light $q \bar{q}$ mesons and $q q q$ baryons. Assuming (and arguing in favour of) the diquark-antiquark-diquark $([u d] \bar{s}[u d]$ ) clustering of this orbitally excited object we found its mass about $290 \mathrm{MeV}$ above the experimental value $1540 \mathrm{MeV}$. In the model considered this discrepancy could be attributed to the change of the constituent mass of $\bar{s}$ antiquark as compared to that of $s$ quark localized at the string end.
\end{abstract}

PACS numbers: 12.38.Lg, 12.39.Mk, 12.40 Yx

The pentaquark $\Theta^{+}(1540)$ was initially predicted in chiral soliton model [1] and then experimentally found by LEPS [2] and DIANA 3] collaborations. Further it was confirmed by many other collaborations $4,5,6,6,7,8,9$, 10]. More than 150 theoretical works are devoted to this subject. Since the pentaquark's chiral soliton quantum numbers are $J^{P}=1 / 2^{+}$, in the quark model it could correspond to the first orbital excitation of five quark system $u u d d \bar{s}$ needed to compensate the negative parity of strange antiquark. The clustering of quarks in five quark system $u u d d \bar{s}$ to two spin-isospin singlet diquarks $[u d][u d]$ and strange antiquark $\bar{s}$ suggested in [1] uses this orbital excitation to assure the Bose statistics for $[u d]$ diquarks that have antisymmetric color function. So, in 11] the pentaquark is considered consisting from two diquarks in relative $p$-wave and strange antiquark in $s$ -wave with respect to the center of mass of two diquarks. It is a first orbital excitation of $[u d] \bar{s}[u d]$ system that has no ground (not orbitally excited) state due to Bose statistics. We will consider the prediction for the mass of this object in the string model.

It is far known (see e.g. the review [12] and references therein) that orbital excitations of light hadrons i.e. of particles consisting from light $u, d, s$ quarks are well described by linear Regge trajectories

$$
J=\alpha^{\prime} M^{2}+\text { const }
$$

with $J$ and $M$ being the spin and the mass of the particle, respectively and $\alpha^{\prime} \approx 1 \mathrm{GeV}^{-2}$ being the slope parameter.

The linear relation between the particle's spin and the mass squared is a result of relativistic string (gluoelectric flux tube) 13] that is formed between light (i.e. with masses small in comparison to string parameter $\alpha^{\prime-1 / 2} \approx$ $1 \mathrm{GeV}$ ) quark clusters sitting at the ends of the string. The string tension $\nu$ and the Regge slope parameter $\alpha^{\prime}$ are related by the formula

$$
\alpha^{\prime}=\frac{1}{2 \pi \nu} .
$$

In the real calculations of the masses of orbitally excited hadrons [12] the asymptotic linear relation between the spin and the mass squared (11) starts practically from the first orbital excitation. The principle ingredient of the string model used in 12] was the spin-orbit coupling provided by Thomas precession. The latter is also connected to the Lorentz-scalar character of the forces responsible for confinement 14]. From experimental point of view there is no systematic spin-orbit interaction for low orbital excitations. This could be the result of cancelation between spin-orbit coupling originating from Lorentz-scalar confining forces and that from Coulomb forces 14]. If one neglect the spin-orbit coupling the spin $J$ and the mass $M$ of the particle lying on the leading Regge trajectory can be found from the following formulae representing the ideal relativistic string with pointlike masses sitting at its ends

$$
\begin{aligned}
J= & \sum_{i} \frac{\nu}{2 \omega^{2}}\left(\arcsin \left(v_{i}\right)-v_{i} \sqrt{1-v_{i}^{2}}\right) \\
+ & \sum_{i} \frac{m_{i} v_{i}^{2}}{\omega \sqrt{1-v_{i}^{2}}}+\sum_{i} s_{i} \\
M= & \sum_{i} \frac{\nu}{\omega} \arcsin \left(v_{i}\right)+\sum_{i} \frac{m_{i}}{\sqrt{1-v_{i}^{2}}}, \\
& \frac{m_{i} v_{i} \omega}{\sqrt{1-v_{i}^{2}}}=\nu \sqrt{1-v_{i}^{2}}
\end{aligned}
$$

where $\omega$ is the angular velocity of rotation, $v_{i}, m_{i}, s_{i}$ are the velocity, mass and spin of the quark cluster at the end of the string, respectively.

The masses of the quark clusters sitting at the ends of the string can be fitted by $\omega-f, K^{*}, \phi, \Lambda$ - trajectories (see the fits on Figs.1-4)

$$
m_{u(d)}=330 \mathrm{MeV}, \mathrm{m}_{\mathrm{s}}=420 \mathrm{MeV}, \mathrm{m}_{[\mathrm{ud}]}=350 \mathrm{MeV} .
$$

At zero orbital angular momentum $\left(v_{i} \rightarrow 0, \omega \rightarrow \infty\right)$ there is no string at all and the model mass $M$ is simply the sum of the masses of quark clusters. As is seen from Figs. 1-4 this is not what we have experimentally and the repulsive interaction of clusters should be included. 


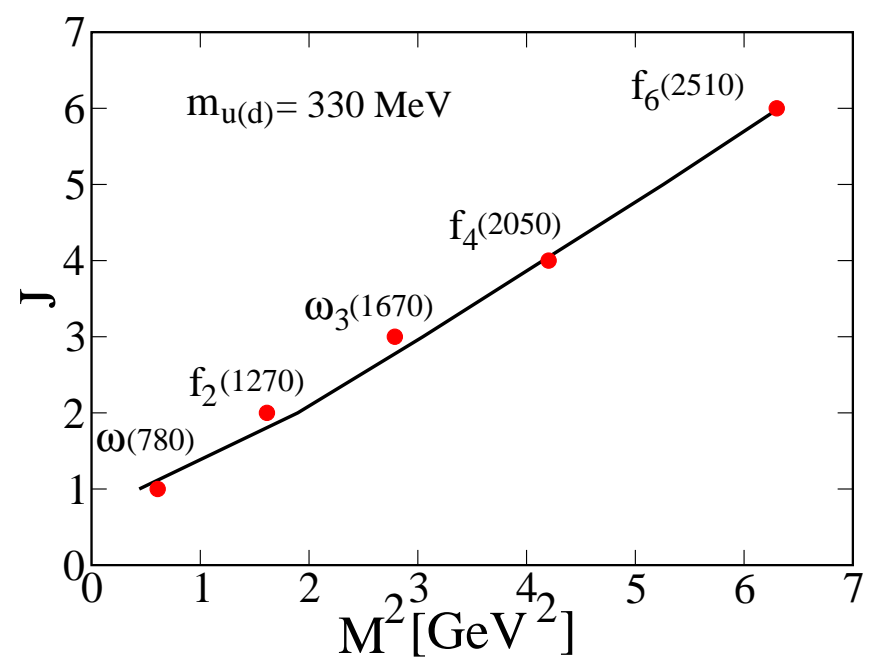

FIG. 1: The leading $\omega-f$ trajectory fitted by the relativistic string with nonstrange quarks at the string ends

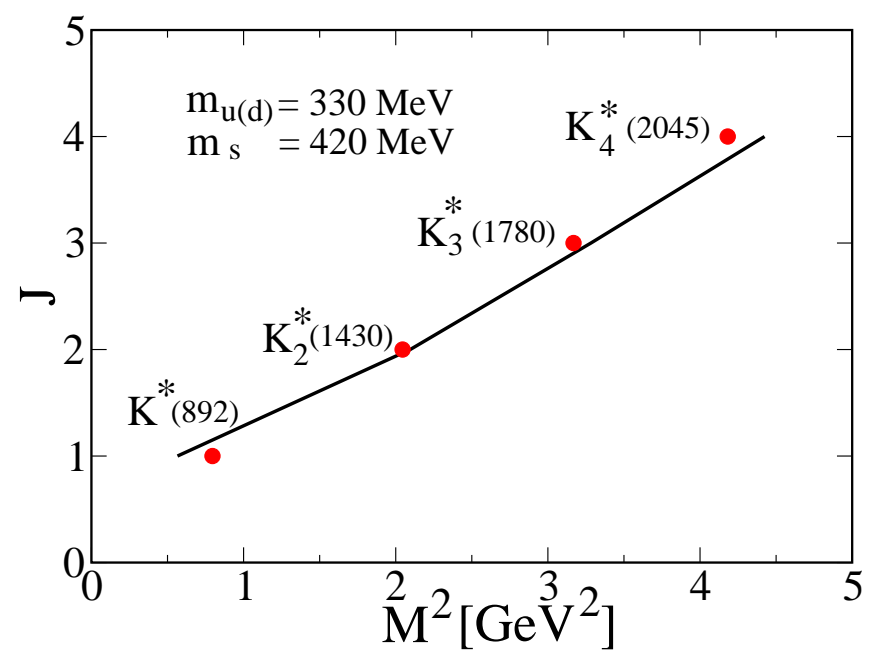

FIG. 2: The leading $K^{*}$ trajectory fitted by the relativistic string with nonstrange + strange quarks at the string ends

Turning now to the pentaquark case and considering it as the first orbital excitation of $[u d][u d] \bar{s}$ system we could imagine at least three configurations for this excitation (see cases (A), (B) and (C) on Fig.5). As for the configurations (A) and (B) the masses of clusters are here fixed from the above considerations of Regge trajectories although in the configuration (B) the strange antiquark is localized not at the end of the rotating string but in the centre at rest, where its localization conditions and, hence, the constituent mass could be different.

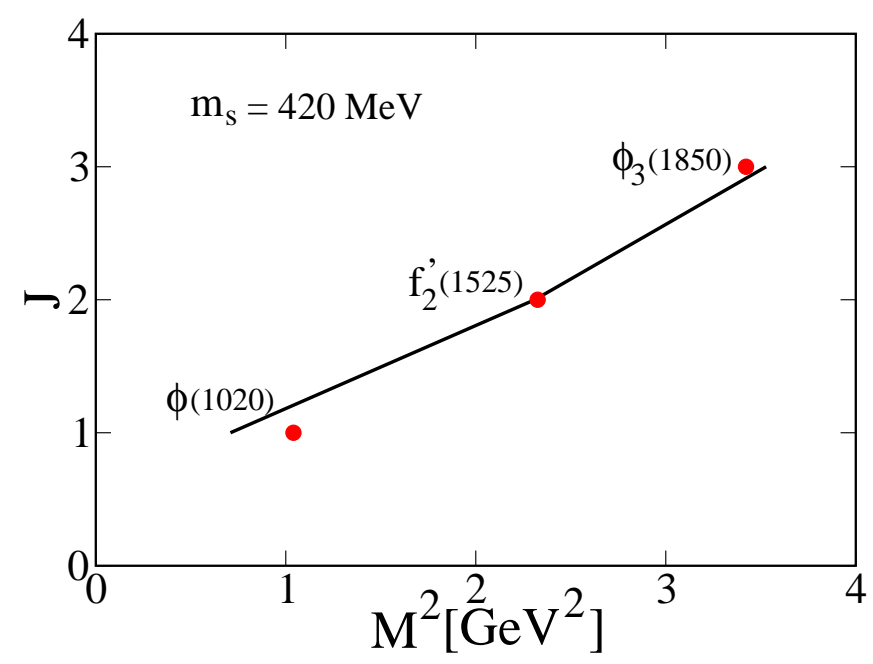

FIG. 3: Model predictions for the leading $\phi$ trajectory by the relativistic string with strange quarks at the string ends

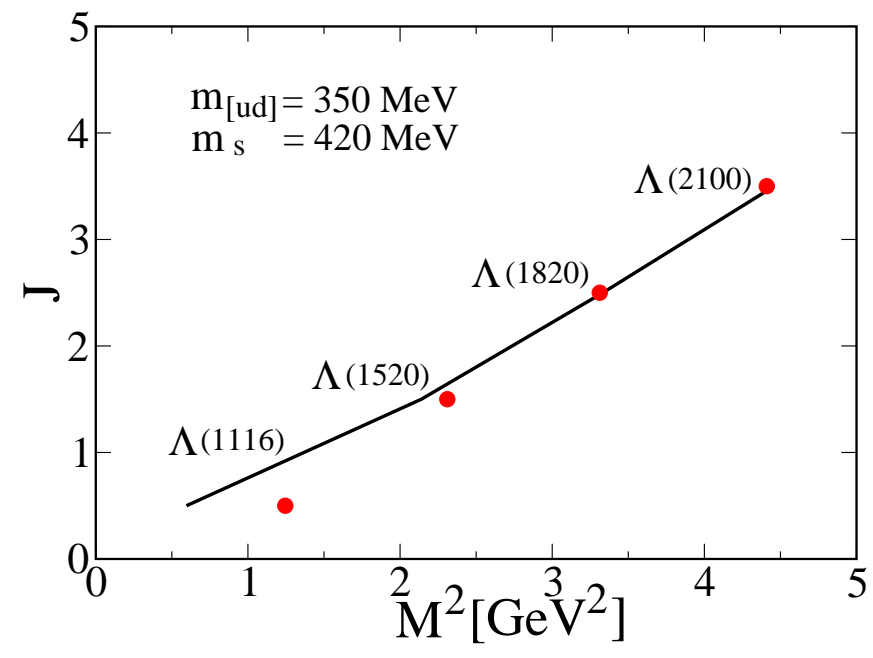

FIG. 4: The leading $\Lambda$ trajectory fitted by the relativistic string with spin-isospin singlet diquark + strange quark at the string ends

The masses of the configurations (A) and (B) are determined by the string dynamics (in the case (B) the mass of strange antiquark is simply added to the mass of the rotating string in eq. (4) ) and are equal to $1930 \mathrm{MeV}$ in case (A) and to $1830 \mathrm{MeV}$ in case (B). By comparing these masses one can conclude that configuration (A) is unstable with respect to transformation to configuration (B). Normally, as a result of the centrifugal forces, the configuration (B) should also be considered unstable with respect to the transformation to the configuration (C). 
But it is not the case due to the strong repulsion of the antiquark $\bar{s}$ and the diquark $[u d]$. To see this let us compare the $\Lambda(1116)$ hyperon with quark structure $[u d] s$ and the $[u d] \bar{s}$ cluster. From Fig.4 (by comparing the mass of $\Lambda(1116)$ hyperon with the string result $\left.m_{[u d]}+m_{s}\right)$ we see that $[u d]$ and $s$ clusters repel each other and this is not the repulsion due to spin-spin interaction which is absent due to zero spin of $[u d]$ diquark. This repulsion could be the result of the change of the confining conditions (change 15 of the constant $B$ of the MIT bag model [16], for example) for [ud]s system in comparison to those of $[u d]$ and $s$ clusters separately. Obviously, the same repulsion should be present in $[u d] \bar{s}$ cluster. If one takes the mass of $\Lambda(1116)$ hyperon as an estimate for the mass of the $[u d] \bar{s}$ cluster the string dynamics gives the mass $2080 \mathrm{MeV}$ for corresponding orbital excitation (configuration $(\mathrm{C})$ ).

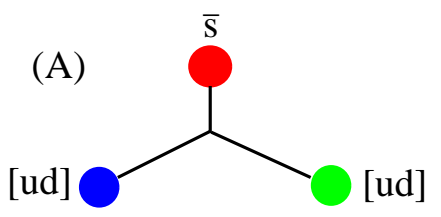

(B)

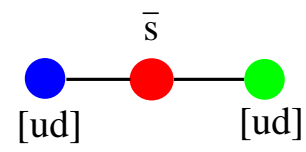

(C)

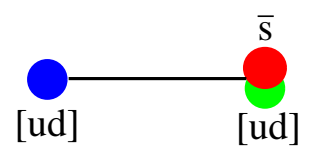

FIG. 5: Possible configurations of the string for $\Theta^{+}(1540)$ pentaquark

So, we consider configuration (B) as the most reliable one for the pentaquark orbitally excited state. Its mass is equal to $1830 \mathrm{MeV}, 290 \mathrm{MeV}$ above the experimental mass. Hence, we are forced to conclude that within the considered string model that properly describes orbital excitations of $q \bar{q}$ and $q q q$ hadrons the orbital excitation of the pentaquark $[u d] \bar{s}[u d]$ is not the $\Theta^{+}(1540)$ particle.

One might ask how reliable is the mass $m_{[u d]}=$ $350 \mathrm{MeV}$ of $[u d]$ diquark extracted from $\Lambda$ hyperon trajectory. If one tries to account for the mass difference of $\rho^{+}(u \bar{d})$ and $\pi^{+}(u \bar{d})$ mesons by the spin-spin quark interaction from gluomagnetic forces i.e. if one writes $m_{\pi}=\left(m_{\pi}+3 m_{\rho}\right) / 4-3\left(m_{\rho}-m_{\pi}\right) / 4$ and $m_{\rho}=$ $\left(m_{\pi}+3 m_{\rho}\right) / 4+\left(m_{\rho}-m_{\pi}\right) / 4$, where the first term is the mass of the object without spin-spin interaction and the second term is the account of spin-spin interaction, then for the mass of spin-isospin singlet diquark we would get $m_{[u d]}=\left(m_{\pi}+3 m_{\rho}\right) / 4-3\left(m_{\rho}-m_{\pi}\right) / 8 \approx 377 \mathrm{MeV}$ because of the spin-spin interaction of $u d$ quarks is two times smaller than the spin-spin interaction of $u \bar{d}$ quarks due to different color functions. This show that our estimation of the $[u d]$ diquark mass is rather reliable.

The mass of the $[u d]$ diquark that reproduces the mass of the $\Theta^{+}(1540)$ in the model considered is equal to $150 \mathrm{MeV}$. Such a light diquark is not compatible with other observations (the $\Lambda$ trajectory and the masses of other hadrons with singlet $[u d]$ diquarks). Nevertheless, it is not excluded by the considerations of dibaryons constructed from three light $[u d]$ diquarks. The reason here is that the (spatial) wave function of three light $[u d]$ diquarks in the dibaryon should be totally antisymmetric what means that one pair of diquarks should have the relative orbital angular momentum equal to one $(l=1)$ and the relative orbital angular momentum of the center of mass of the first pair and third diquark should also be equal to one $(L=1)$. In total these angular momenta should be summed again to one $(l+L=1)$. This is the lowest energy state of three diquarks allowed by Bose symmetry (with deuteron quantum numbers). Its totally antisymmetric spatial wave function has the form $\left(\overrightarrow{x_{1}} \times \overrightarrow{x_{2}}+\overrightarrow{x_{2}} \times \overrightarrow{x_{3}}+\overrightarrow{x_{3}} \times \overrightarrow{x_{1}}\right) f\left(\overrightarrow{x_{1}}, \overrightarrow{x_{2}}, \overrightarrow{x_{3}}\right)$ where $f\left(\overrightarrow{x_{1}}, \overrightarrow{x_{2}}, \overrightarrow{x_{3}}\right)$ is the totally symmetric under permutations of coordinates function. We don't know which semiclassical configuration of rotating strings could correspond to the above wave function but assume that $l+L=2$ configuration, which is different from $l+L=1$ configuration only by the way how two units of orbital angular momenta are summed in the total orbital angular momentum and semiclassically corresponds to the rotating starlike object (see Fig.5(A) with $\bar{s}$ substituted by $[u d]$ ), has the same energy. The latter can be computed by string dynamics. For the mass of $[u d]$ diquark needed for the reproduction of the mass of $\Theta^{+}(1540)\left(m_{[u d]}=150 \mathrm{MeV}\right)$ the mass of the dibaryon with deuteron quantum numbers is equal to $1910 \mathrm{MeV}$. It is $30 \mathrm{MeV}$ above the protonneutron threshold and the dibaryon is expected to be as narrow as the $\Theta^{+}(1540)$ pentaquark. Such narrow deuteron-like dibaryon is not observed.

In conclusion, in the simple string model for orbital excitations of light hadrons with the masses of constituent $[u d]$ diquark and $s$ quark found by fitting $\omega, K^{*}, \phi, \Lambda$ trajectories the mass of the $\Theta^{+}(1540)$ pentaquark, considered as a first orbital excitation of the $[u d] \bar{s}[u d]$ system, is $290 \mathrm{MeV}$ above the experimental value. As have already been discussed above in the configuration (B) the strange antiquark is localized not at the end of the rotating string but in the centre at rest, where its localization conditions and, hence, constituent mass could be different. So, in the model considered the observed discrepancy could be attributed to the change (the lowering) of the constituent mass of $\bar{s}$ antiquark as compared to that of $s$ quark localized at the string end.

This work is supported by RFBR grant No. 03-0204004, DFG grant No. 436 RUS 113/721/0-1, and by Federal Program of the Russian Ministry of Industry, Science and Technology No. 40.052.1.1.1112. M.I.K. and B.V.M. acknowledge the kind hospitality at the University of Tuebingen. 
[1] D. Diakonov, V. Petrov, and M. Polyakov, Z. Phys. A 359, 305 (1997).

[2] LEPS Coll., T. Nakano et al., Phys. Rev. Lett. 91, 012002 (2003).

[3] DIANA Coll., V. V. Barmin et al., Phys. At. Nucl. 66, 1715 (2003).

[4] CLAS Coll., S. Stepanyan et al., Phys. Rev. Lett. 91, 252001 (2003).

[5] CLAS Coll., V. Kubarovsky et al., Phys. Rev. Lett. 92, 032001 (2004).

[6] SAPHIR Coll., J. Barth et al., Phys. Lett. B 572, 127 (2003).

[7] HERMES Coll., A. Airapetian et al., hep-ex/0312044

[8] SVD Coll., A. Aleev et al., hep-ex/0401024
[9] COSY-TOF Coll., M. Abdel-Bary et al., hep-ex/0403011

[10] A. E. Asratyan, A. G. Dolgolenko, and M. A. Kubantsev, hep-ex/0309042

[11] R. Jaffe and F. Wilczek, Phys. Rev. Lett. 91, 232003 (2003).

[12] I.Yu. Kobzarev, B.V. Martemyanov, M.G. Shchepkin, Sov.Phys.Usp.35, 257 (1992)

[13] Y. Nambu, Phys.Rev.D10 4262 (1974).

[14] E. Eichten , F. Feinberg, Phys.Rev. D23, 2724 (1981).

[15] I.Yu. Kobzarev, B.V. Martemyanov, M.G. Shchepkin, Sov.J.Nucl.Phys. 29, 831 (1979).

[16] A. Chodos, R.L. Jaffe, K. Johnson, Charles B. Thorn, V.F. Weisskopf, Phys.Rev.D9, 3471 (1974). 